Copyright ( 2013 IEEE. Personal use of this material is permitted. Permission from IEEE must be obtained for all other uses, in any current or future media, including reprinting/republishing this material for advertising or promotional purposes, creating new collective works, for resale or redistribution to servers or lists, or reuse of any copyrighted component of this work in other works. 


\section{On the Effects of Energy-Aware Traffic Engineering on Routing Reliability}

\author{
Gongqi Lin, Sieteng Soh, Mihai Lazarescu \\ Department of Computing \\ Curtin University of Technology \\ Perth Australia \\ Gongqi.lin@postgrad.curtin.edu.au
}

\author{
Kwan-Wu Chin \\ School of Electrical, Computer and Telecommunications \\ Engineering \\ University of Wollongong \\ Wollongong Australia \\ kwanwu@uow.edu.au
}

\begin{abstract}
Current network infrastructures are over-provisioned to increase their resilience against resource failures, $e . g$., bundled links and nodes, as well as congestion during peak hours. However such strategies waste resources as well as exhibit poor energy efficiency at off-peak periods. To this end, several energyaware routing algorithms have been proposed to maximally switch off redundant network resource at low traffic load to minimize energy usage. These routing solutions, however, do not consider network reliability as critical back-off links/nodes maybe switched off. Henceforth, we aim to quantify the effects of five recently proposed green routing approaches, namely FGH, GreenTE, MSPF, SSPF, and TLDP, on the following two reliability measures: (i) 2-terminal reliability (ii) path reliability. Experiments using three topologies with real and synthetic traffic demands show that switching off redundant links significantly affects the 2-terminal reliability. Routing traffic through multiple paths has lesser reliability impact while reducing energy, especially when the paths are link disjoint. Interestingly, TDLP and MSPF have better path reliabilities than using shortest path routing.
\end{abstract}

Keywords - energy-aware routing; network reliability; multiple paths; bundled links; two link disjoint paths; shortest path

\section{INTRODUCTION}

In the past two decades, new and popular Internet applications, such as electronic commerce, voice over IP, social networking, have emerged. In addition, many applications, such as online banking and health care services, are business critical and time sensitive. Therefore, the Internet has become more than just a network used to access information. Indeed, a high degree of network reliability is taken for granted by users and businesses [24]. In this context, network resiliency, i.e., the ability to recover quickly and smoothly from resource failures or disruptions is becoming increasingly important in the operation of modern IP networks. Consequently, commercial carriers, e.g., AT\&T, BT and NTT, routinely set network reliability as a critical performance objective when deploying communication services. This is in addition to network usability and fault processing capabilities [16].

To this end, researchers have proposed using backup paths $[6,7,8]$ or route restoration through traffic engineering $[9,12$, $13,14,24]$ to increase network resiliency. Route restoration, which finds a new route from backup paths that do not contain failed resources, involves tradeoffs between efficient use of network resources and resiliency against failures [25]. Note that backup paths can be generated only when the network has sufficient path diversity, which in turn is only possible when a network is over-provisioned with backup links and/or nodes. While over-provisioning resources to provide backup paths and routing restoration using traffic engineering (TE) help improve reliability, it exhibits poor energy efficiency, especially at low traffic load.

Reducing energy consumption has recently become an important part of networking research, and recent reports $[1,2$, $3,4]$ show that the power consumption of large wired networks has increased tremendously. For example, the United States alone spends nearly $\$ 0.5-2.4$ billions a year on powering wired networks [2]. The authors in [4] projected that even smaller systems such as Akamai consume an estimated $\$ 10 \mathrm{M}$ worth of electricity annually.

To this end, given a set of traffic demand $D$, green-routing mechanisms have been proposed to generate an energy-aware network $G$, with links/nodes from the original network $G$ switched off such that all demands in $D$ are satisfied. Fisher $e t$ al. [17] consider each core router in $G$ connected by multiple physical cables that form one logical bundled link, and propose to turn-off redundant cables. Their solution, called FGH, reroutes traffic demands using only switched-on cables in $G^{\prime}$. This, however, may affect network reliability as compared to using shortest path (SP) routing on the original network $G$. Zhang et al. [18] observed that network operators usually provide redundant network links and set a link utilization bound, e.g., $40 \%$, to ensure their networks are fault-tolerant against network failures and congestion. They propose a power-aware traffic engineering algorithm, called GreenTE, to maximally switch off links while satisfying two performance constraints: maximum link utilization (MLU) and path hop count. GreenTE aims to produce $G$ ' by turning off the maximum number of links from $G$ such that all traffic in $D$ are satisfied, each link has utilization no more than $50 \%$, and each demand is re-routed through one or more paths with hop counts no longer than a given constraint. The MSPF solution [26] generalizes FGH and GreenTE; it considers bundled links similar to FGH as well as the two constraints used in GreenTE. 
Lin et al. [27] propose another TE solution, called SSPF, which re-route each demand using only a single path. They show that single path routing is attractive for its simplicity as well as fast route and significant energy saving. In [28], when a demand cannot be rerouted using a single path, the authors propose to reroute it using two-link disjoint paths to improve its throughput and fault tolerance. Note that the multiple paths used in FGH, GreenTE and MSPF $[17,18,26]$ may not be linkdisjoint. While all solutions $[17,18,26,27,28]$ are able to turnoff significant number of redundant links/cables to reduce power consumption, none of them reported their impact on network reliability.

Henceforth, this paper studies the impact of switching off cables using five recent energy-aware routing mechanisms, namely FGH, GreenTE, MSPF, SSPF, and TLDP [17, 18, 26, 27,28 ], on two reliability measures, i.e., 2-terminal reliability and path reliability. The first reliability measures the probability of obtaining at least one operational path that can be used for route restoration in case of link failures, while the second computes the reliability of each path used to route traffic. To the best of our knowledge, this work is the first to evaluate the effects of switching off network resources to save energy on the network and path. Our study involving three real topologies, namely Abilene, GÉANT and Sprint using both real and traffic demands highlight show that the energy-aware routings have negative impacts on 2-terminal reliability of the topologies with switched-off cables. Interestingly, MSPF and TLDP produce paths with reliability higher than via shortest paths on the original networks. We believe the results presented in this paper hold for other energy-aware routing algorithms.

The rest of the paper is organized as follows. Section II describes the network model, our notations, and two reliability measures. Section III gives an overview of the problem and its linear programming formulation, and compares the constraints of five energy-aware routing solutions. Section IV evaluates the reliability and energy savings for five state-of-art algorithms using both real and synthetic topologies and data. Finally, Section V concludes the paper.

\section{BACKGROUND}

\section{A. Network Model and Notations}

Consider a computer network modeled by a directed graph $G(V, E)$, where $V$ is the set of $n$ nodes, and $E$ is the set of $m$ links. Each node represents a router and each link $(i, j)$ between nodes $i$ and $j$ represents a bundled link as a communication channel with a limited capacity/bandwidth $c_{i j}>0$. Each link $(i, j)$ consists of $w_{i j} \geq 1$ cables. We call $w_{i j}$ the bundle size of link $(i, j)$. Each cable can be switched-off independently. Assume each cable in $(i, j)$ has the same bandwidth and power consumption. Let $n_{i j} \leq w_{i j}$ be an integer that represents the total number of powered-on cables in $(i, j)$; link $(i, j)$ is switched off when $n_{i j}=0$.

Each cable in $(i, j)$ has a probability $0 \leq r_{i j} \leq 1$ of being functional We assume all nodes are always functional, and cable failures are statistically independent with equal probability. Since each link $(i, j)$ is comprised of parallel cables, the reliability of $\operatorname{link}(i, j)$ can be computed as $1-\left(1-r_{i j}\right)^{n_{i j}}$.
Let $D$ be a set of all demands in $G(V, E)$, and $\left(s_{d}, t_{d}, b_{d}\right)$ denote a traffic demand with $d=1,2, \ldots,|D|$ between source node $s_{d} \in V$ and terminal node $t_{d} \in V$, where $b_{d}$ is the throughput between $s_{d}$ and $t_{d}$. Let $P_{d}$ be a set of $\left(s_{d}, t_{d}\right)$ paths, each of which has delay $h_{d}$ no more than a given threshold $T_{d}$. In practice, network operators can have their own definitions of link delays and path lengths, and choose the set of candidate paths accordingly. Each $h_{d}$ is defined as link propagation delays of path for each $\left(s_{d}, t_{d}\right)$; in other words, we add up link propagation delays to get path lengths. Further, when a traffic demand $d$ is routed through multiple $\left(s_{d}, t_{d}\right)$ paths $M P_{d} \subseteq P_{d}$, we set its path delay $h_{d}$ to the maximum link propagation delays of paths in $M P_{d}$. We denote $f_{i j}^{d}$ as the flow on link $(i, j)$ of demand $d$, and $f_{i j}$ the total flow on link $(i, j)$. Finally, the link utilization of $(i, j)$ is computed as $u_{i j}=f_{i j} / c_{i j} * 100 \%$.

\section{B. Reliability Computation}

For each pair of source node $s_{d}$ and terminal node $t_{d}$, this paper considers two reliability measures: (i) two-terminal reliability, and (ii) path reliability. Computing the former is a well known NP-hard problem [10]. The reliability is defined as the probability that there exists at least one operational $\left(s_{d}, t_{d}\right)$ path between the source and terminal node. Many solutions have been proposed in the literature to compute the reliability. In this paper, we use CAREL [10] to compute the reliability, $R_{d}$, for each $\left(s_{d}, t_{d}\right)$ demand in $D$. Each $R_{d}$ for demand $d$ is calculated in two steps. First, we use Yen's algorithm [5] to generate all possible $\left(s_{d}, t_{d}\right)$ paths. Then, we use CAREL to compute $R_{d}$ from the path set. Interested reader can see the details of CAREL in [10].

The measure in (ii) computes the reliability of $\left(s_{d}, t_{d}\right)$ path used to route demand $d$. For single path routing, as carried out by SSPF and SP, one computes the measure by multiplying the reliability of each link in the path. Two link disjoint paths, produced by TLDP [28], can be computed as the reliability of a two parallel system [15]. Specifically, for two link disjoint paths, each with reliability $\rho_{1}$ and $\rho_{2}$ respectively, we compute their reliability as $1-\left(1-\rho_{1}\right)\left(1-\rho_{2}\right)$. This paper uses CAREL [10], to compute the reliability of each non-disjoint multiple paths that can be produced by MSPF, FGH, GreenTE, and TLDP.

\section{GREEN ROUTING ALGORITHM COMPARISONS}

In this section, we first state the general problem addressed by five algorithms. Then, we discuss four main differences among the five green routing heuristic approaches.

\section{A. Problem Statement}

Given a network $G(V, E)$ and a traffic demand set $D$, the optimization problem in MSPF is to generate (i) the minimum number of powered on cables, and (ii) the set of paths $P_{D}=\left\{M P_{d}|d=1,2, \ldots| D \mid,\right\}$ that can be used to route traffic of each demand $d \in D$ while using only powered-on cables, subject to two constraints: $(\mathrm{C} 1)$ the utilization of each link $(i, j)$ is no larger than a given threshold $U_{T}$, i.e., $u_{i j} \leq U_{T}$, and $(\mathrm{C} 2)$ the length of each path in $M P_{d}$ for demand $d$ is no longer than a 
given constraint $T_{d}$, i.e., $h_{d} \leq T_{d}$. In other words, the problem is to find as many cables that can be switched off while satisfying all traffic demands in $D$ under constraints (C1) and (C2).

The two constraints are used to ensure the solution, while minimizing energy usage, does not affect the QoS of customers. In particular, we note that the average link utilization in backbone networks of large ISPs is deliberately set to around $30-40 \%$ in order to guarantee QoS [17]. The problem can be formulated as a mixed integer programming (MIP) as follows.

Minimize:

$\sum_{(i, j) \in E} n_{i j}$

Subject to:

$$
\begin{aligned}
& \sum_{j \in V} f_{i j}^{d}-\sum_{j \in V} f_{j i}^{d}=\left\{\begin{array}{c}
b_{d}, i=s_{d} \\
-b_{d}, i=t_{d} \\
0, \text { Otherwise }
\end{array}, \forall d \in D\right. \\
& f_{i j}=\sum_{d \in D} f_{i j}^{d} \leq U_{T}\left(n_{i j} / w_{i j}\right) c_{i j}, \forall(i, j) \in E \\
& 0<h_{d} \leq T_{d}, \forall d \in D \\
& 0 \leq n_{i j} \leq w_{i j}, \forall(i, j) \in E
\end{aligned}
$$

Equation (1) quantifies the total number of powered-on cables, and thus power consumed by all active cables. Equation (2) is the standard flow conservation constraint that ensures no flow is lost, and ensures that the sum of the flows leaving the source, or entering the destination of demand $d$ sums to $b_{d}$. Equation (3) computes the total flow traversing each link while restricting it to within the link's allowable capacity utilization, i.e., $U_{T}\left(n_{i j} / w_{i j}\right) c_{i j}$. Equation (4) restricts each path delay $h_{d}$ to be longer than 0 since it needs at least one link to transmit each traffic demand and no longer than a given delay constraint $T_{d}$. Constraint (5) bounds the number of active cables to be less than the bundle size of each link. The resulting formulation is a MIP problem, which is generally NP-hard [18], due to the integer variable $n_{i j}$. Thus its computation time for networks with medium and large sizes is still a challenge issue.

\section{B. Differences among Green Routing Algorithms}

We observe four main differences between FGH, GreenTE, MSPF, SSPF, and TLDP, namely, bundle size, QoS constraints, the number of paths used to route each demand, and how each path to route each demand is generated. The details of each difference are as follows.

\section{1) Bundle Links}

The use of bundled links provide more flexibility with regards to increasing link capacity as well as reliability, and thus support network resiliency in case of cable failures and congestion. Moreover, each cable is assumed to have the same bandwidth and reliability, meaning a larger bundle size increases link bandwidth as well as reliability. FGH, MSPF, SSPF, and TLDP consider each link to have $w_{i j} \geq 1$ cable(s), and thus affords the said benefits as compared to GreenTE, which uses $w_{i j}=1$. In other words, when GreenTE switches off a link, the entire line-card would be put to sleep, and has less flexibility in rerouting traffics since it cannot switch off each cable in a link independently. With respect to the MIP formulation, GreenTE sets $w_{i j}=1$ in Eq. (3) and (5).

\section{2) QoS Constraints}

GreenTE and MSPF aim to route all demands while satisfying the two QoS constraints, i.e., maximum link utilization and path hop counts, specified in Eq. (3) and (4), respectively. In contrast, FGH does not require any of the two constraints, and thus it excludes Eq. (4) and sets $U_{T}=100 \%$ in Eq. (3). Both SSPF and TLDP consider only the maximum link utilization constraint, and thus $T_{d}=\infty$ in constraint (4). With respect to reliability, including the QoS constraints will have a negative effect since the constraints limit available paths that can be used for route restoration.

\section{3) Single Path vs. Multiple Paths}

Multipath routing provides additional resiliency by providing fast (or simultaneous) access to backup paths. GreenTE, FGH, MSPF and TLDP allow each demand to be routed through multiple paths while SSPF restricts each demand through a single path; thus SSPF routing is the least reliable. Note that in most router implementations, packets that belong to a particular TCP session (i.e., going to a specific destination in terms of IP address) are routed on a specific shortest-path (even if multiple shortest paths are available). In contrast, TLDP aims to reroute each demand through two linkdisjoint paths, whenever possible. Protecting a connection over link-disjoint paths has the obvious advantage of better fault tolerance, which can enhance network reliability; thus TLDP offers the most reliable routing as compared to the other four schemes. With respect to the MIP, for SSPF, the flow in (2) is routed through only one path. Specifically, Equation (2) is replaced with the following constraint:

$$
\sum_{(i, j) \in E} x_{i j}^{d}-\sum_{(j, i) \in E} x_{j i}^{d}=\left\{\begin{array}{c}
1, \quad i=s_{d} \\
-1, \quad i=t_{d} \quad, \quad \text { Otherwise }
\end{array}, \forall i \in V, d \in D\right.
$$

where $x_{i j}^{d}$ is a binary variable that is set to $1(0)$ when the traffic $d$ is routed (not routed) through link $(i, j)$; see [26] for more detail explanation. With respect to the MIP, TLDP ignores constraint (4), but includes constraint $T_{\max }-$ the percentage of the total number of paths that have at least one two link-disjoint paths. This additional constraint provides routing service with higher fault-tolerance and bandwidth to upper layer applications.

\section{4) Path Selections}

FGH and GreenTE use LP solver CPLEX [19] to generate paths for all demands after switching off cables/links, and therefore the algorithms cannot explicitly select more reliable paths for a set of demands. Consequently, either FGH or GreenTE cannot be used for applications that require paths with reliability constraints. In contrast, SSPF, MSPF, TLDP generate their paths for each demand $d$ in two steps. First, they use Yen's algorithm [5] to generate the first $k\left(s_{d}, t_{d}\right)$ shortest paths. Then SSPF (TLDP) selects the shortest (two link-disjoint) path (paths) from the $k$ paths that satisfies maximum link utilization requirement, while MSPF selects multiple paths, 
starting from the shortest, that satisfy both link utilization and path delay constraints. Therefore, one can directly extend each of the three algorithms for use in applications that require path with reliability constraints,.

\section{Evaluation}

In this section, we compare the effects of using FGH, GreenTE, MSPF, SSPF and TLDP on the two reliability measures, namely 2-terminal reliability and path reliability, as well as power savings. In this evaluation, we set the parameters in the general problem formulation, shown in Section III.A, as follows: i) bundle size $w_{i j}=1$ since GreenTE does not support bundled links, ii) a threshold of path delay $T_{d}=\infty$ because FGH, SSPF and TLDP do not consider path length constraint, iii) a threshold of MLU $U_{T}=100 \%$ since FGH also ignores link utilization constraint. Further, we set $k=100$ for GreenTE, SSPF and MSPF, and assume equal cable reliability $r_{i j}=0.9$.

For each topology $G$, we use each algorithm to switch off redundant cables to generate $G^{\prime}$. Then we used CAREL [10] to compute 2-terminal reliability $R_{d}$ from $G$ ' for each demand $d \in D$. Further, we also calculate path reliability on each demand using the method described in Section II.B. We compute the average 2-terminal reliability, AR, as follows:

$$
A R=\sum_{d \in D} R_{d} /|D|
$$

We compute each network's power saving, PS, using the following formula:

$$
P S=\left(1-\frac{\sum_{(i, j) \in E} n_{i j}}{\sum_{(i, j) \in E} w_{i j}}\right) \times 100 \%
$$

Note that $\sum_{(i, j) \in E} w_{i j}=m$ since each $w_{i j}=1$. We compute the average 2-terminal reliability and the reliability of paths, for each demand $d \in D$, generated by $\mathrm{SP}$ routing on original network $G$ as benchmarks for (7) and (8) respectively.

\section{A. Experiment Setup}

As shown in Table II, our experiments used three real topologies, i.e., Abilene [21], GÉANT [20] and Sprint [22].

TABLE I. NETWORK TOPOLOGIES

\begin{tabular}{|c|c|c|c|c|}
\hline Network & Usage & Location & Nodes & Links \\
\hline Abilene & Research & US & 12 & 30 \\
\hline GÉANT & Research & Europe & 23 & 74 \\
\hline Sprint & Commercial & US & 52 & 168 \\
\hline
\end{tabular}

For the Abilene topology, we use the 288 traffic matrices measured on September 5, 2004 for every five minutes for duration of 24 hours - all of which are provided by the authors of [21]. For GÉANT, its traffic matrix was collected on May 5, 2005 [22]. For Sprint, we randomly generate a traffic matrix using the gravity model [18], and scaled the traffic to obtain 40 different traffic matrices. Specifically, the generated traffic matrices is such that when traffic is routed using the SP, the maximum link utilization of the topology is $2.5 \%, 5 \%, 7.5 \%$, $\ldots, 97.5 \%, 100 \%$. In this paper, we refer the traffic matrix that results in a MLU of $2.5 \%$ using SP routing as MLU $2.5 \%$ under SP, MLU of 50\% as MLU 50\% under SP, etc. All nodes in these three topologies are access nodes, which also act as transit (backbone or core) nodes to route multi-hop traffic demands. For these topologies, consistent with the traffics used in [21] and [22], we consider all possible end-to-end traffic demands, i.e., each node is the traffic source/sink. Consequently, none of the access nodes can be switched off. Our simulations were performed on a Linux PC with $3.07 \mathrm{GHz}$ CPU and 8GB RAM. For GreenTE [18] and FGH [17], we ran the source code provided by their respective authors, and the CPLEX [19] LP solver.

\section{B. Effects on 2-Terminal Reliability}

\section{1) Abilene}

Fig. 1 and Fig. 2 show the average 2-terminal reliability of all traffic and the power saving produced by all routing algorithms on Abilene network, respectively. Among the energy-aware routers, Fig. 1 shows that TLDP produces the best reliability among the energy aware routers with $\mathrm{AR}=0.846$, but it reduces power usage only $20 \%$, the worst performer. Note that TDLP re-routes each traffic demand through two link-disjoint paths whenever possible and thus increases network fault tolerance. In contrast, SSPF is the best performer on power savings, shutting down 56.7\% total cables, but the worst performer with reliability with an AR of 0.582 because it uses single path routing. Note that SP also uses a single path routing; however it uses the shortest path for each traffic, and therefore has the best reliability but has no power saving. In addition, MSPF has higher reliability than FGH, which has an AR value of 0.682 against 0.612 , although they have the same energy savings. Fig. 2 shows the power savings for Abilene network using four heuristics. As shown in the figure, while effective in reducing energy, SSPF and GreenTE result in the lowest reliability, at $\mathrm{AR}=0.582$.

\section{2) Sprint}

Fig. 3 and Fig. 4 show the average reliability and power savings for FGH, GreenTE, SSPF, MSPF, and TLDP on Sprint's network, respectively. For Sprint with MLU 100\% under SP, TLDP produces the highest reliability of 0.905 , only $7.8 \%$ lower than original network with $\mathrm{AR}=0.981$, while saving $19.23 \%$ power usage. The figures show MSPF as the best overall performer, achieving reliability of 0.75 , while saving the most power, i.e., PS $=48.7 \%$, with GreenTE as the second best. Overall, while saving power for up to $46.7 \%$, SSPF, GreenTE and FGH reduces the network reliability by up to $40.8 \%$. The results further confirm that energy aware routings, while able to switch off redundant cables, significantly reduce network's reliability.

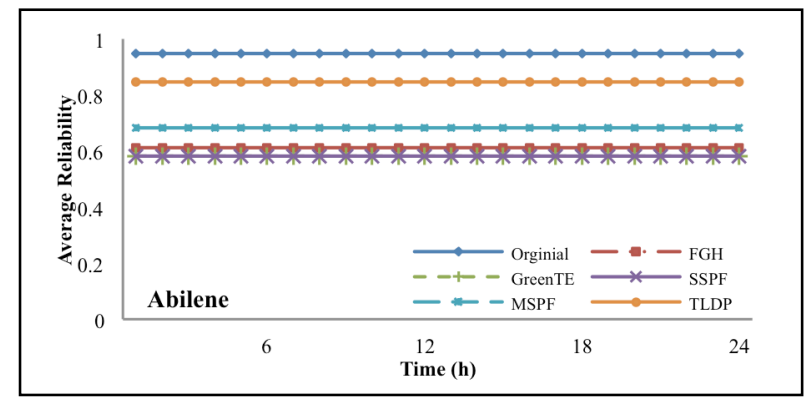

Figure 1. Average Reliability on Abilene Topology 


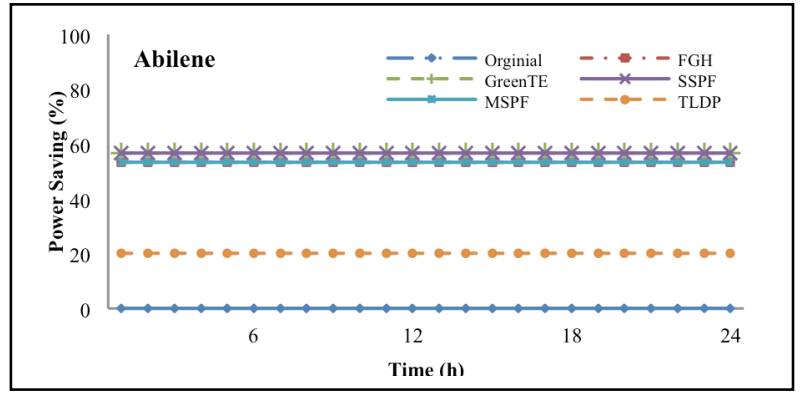

Figure 2. Power Saving on Abilene Topology

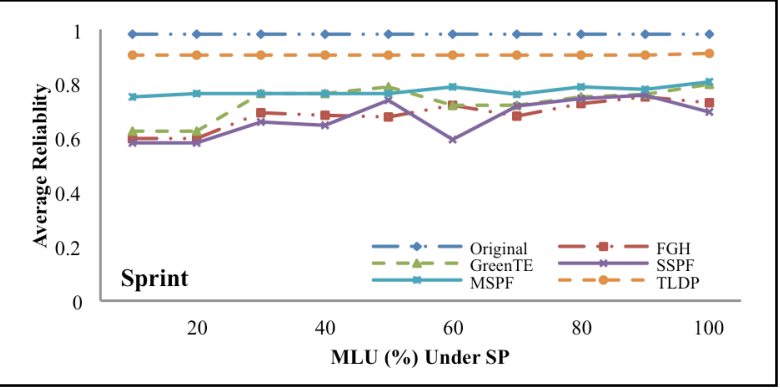

Figure 3. Average Reliability on SprintTopology

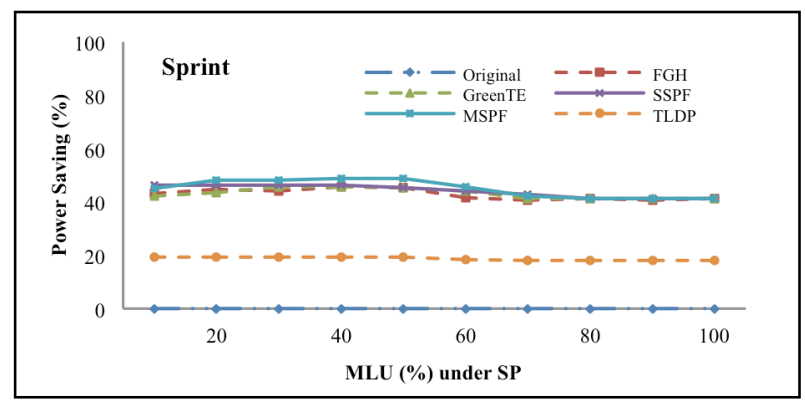

Figure 4. Power Savings on Sprint Topology

\section{3) GÉANT}

Table II shows the effects of saving power on the average reliability of the GÉANT network. From the table, we see TLDP reduces reliability only by $2.6 \%$, the best performer, while able to save $24.3 \%$ of power. Among the other schemes, MSPF is arguably the best performer since it has the second highest reliability (0.748) while able to reduce energy among the highest $(58.1 \%)$. The result is consistent with those for Abilene and Sprint. Notice that SSPF and FGH perform best on the power saving, at $58.5 \%$, but they reduce network's reliability by $38 \%$, the worst performer with $\mathrm{AR}=0.612$.

TABLE II. COMPARISION ON GÉANT NETWORK

\begin{tabular}{|c|c|c|}
\hline Methods & AR & PS (\%) \\
\hline Original & 0.987 & 0 \\
\hline FGH & 0.612 & 58.5 \\
\hline GreenTE & 0.728 & 58.1 \\
\hline SSPF & 0.612 & 58.5 \\
\hline MSPF & 0.748 & 58.1 \\
\hline TLDP & 0.961 & 24.3 \\
\hline
\end{tabular}

\section{Effects on Path Reliability}

Fig. 5, 6 and 7 show the Cumulative Distribution Function (CDF) of path reliability of using the five energy-aware routing approaches, and Shortest Path (SP) routing without considering energy savings for Abilene, GÉANT and Sprint networks respectively. The figures show, interestingly, that both TLDP and MSPF improve the path reliability of the SP while reducing energy usage. In particular, $94 \%, 37 \%$ and $52 \%(65 \%, 22 \%$ and $37 \%)$ of paths produced by TDLP (MSPF) for Abilene, GÉANT and Sprint have paths with reliability at least $0.8,0.9,0.8$ respectively, significantly better than only $55 \%, 0 \%$ and $26 \%$ by SP. In term of path reliability, TLDP is the best since it reroutes each demand via two linkdisjoint paths if possible; thus TDLP is suitable for applications that require high reliability.

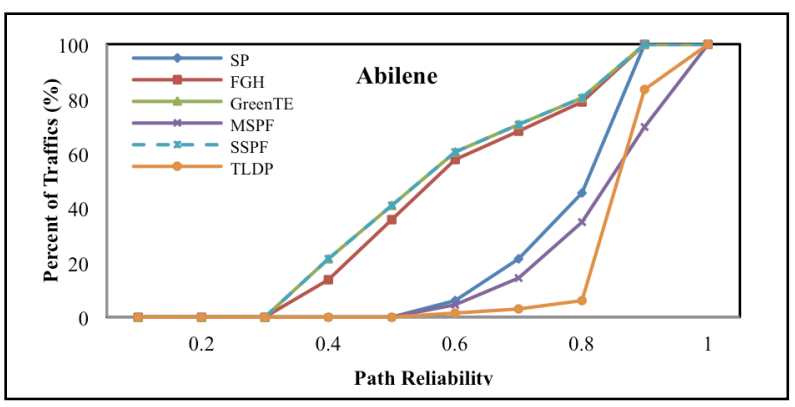

Figure 5. CDF of Path Reliability on Abilene

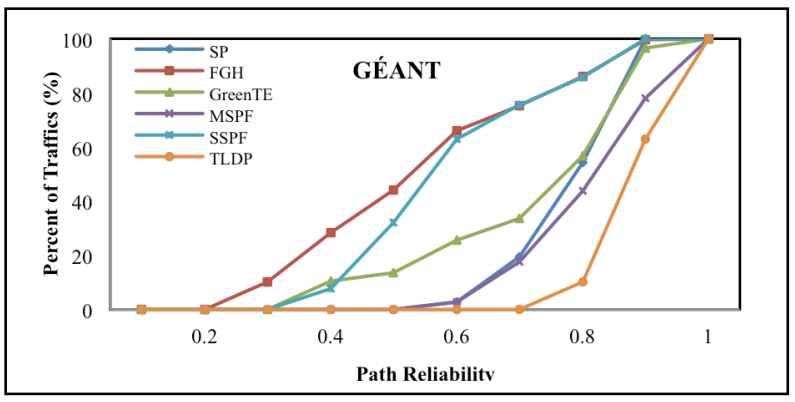

Figure 6. CDF of Path Reliability on GÉANT

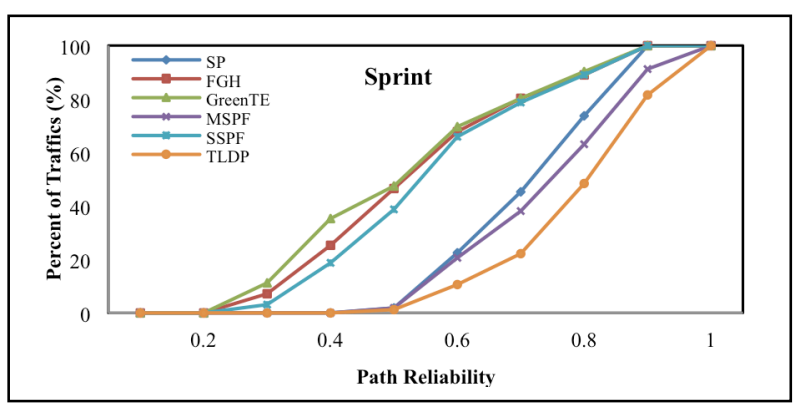

Figure 7. CDF of Path Reliability on Sprint

In contrast, GreenTE, FGH and SSPF consistently perform among the worst on the three networks. Specifically, on Abilene, only $40 \%$ of its paths have reliability larger than 0.6 , none of which has reliability above 0.8 . Note that GreenTE and SSPF have the same worst CDF; FGH is slightly better. For GÉANT, FGH and SSPF are the worst and second worst, producing $65.92 \%$ and $62.8 \%$ traffics that have reliability no 
higher than $60 \%$. For Sprint, $67.72 \%$ and $69.92 \%$ of routes produced by FGH and GreenTE have reliability no larger than $60 \%$. Notice that MSPF consistently produce more reliable routes than GreenTE, FGH, and SSPF on the three networks. Since energy savings using MSPF on the networks are competitive against GreenTE, FGH and SSPF, MSPF performs better than GreenTE, FGH and SSPF.

\section{CONCLUSION}

We have described five different routing algorithms to reduce network energy usage by switching off cables/links at off-peak period. We have discussed differences among the algorithms under four categories, bundle size, path types (single path, two link-disjoint paths and multiple paths), QoS and path selections, and their possible effects on network reliability. Our evaluation on Abilene, GÉANT and Sprint topologies using both real and synthetic traffic shows that the algorithms, while effective in saving energy, significantly reduce the networks' 2-terminal reliability, meaning they reduce the possibility of path restoration in the event of link failures. Consistent with our analytical comparison, TLDP has the least effect on reliability but reduces energy the least as compared to the other four algorithms. Interestingly, while able to reduce significant energy usage, TDLP and MSPF produce paths with better reliability as compared to using shortest path routing. We found that MSPF strikes the best balance between reducing energy and negative effect on reliability. As compared to FGH and GreenTE that use an LP solver to reroute traffic, MSPF, SSPF, and TLDP are more flexible in rerouting paths, and thus can be extended for applications that require paths with reliability constraints. In the future, we plan to extend each of the last three algorithms such that they can generate routes with lesser effect on reliability while maintaining their effectiveness in reducing energy usage.

\section{ACKNOWLEDGMENT}

We thank M. Zhang and W. Fisher for providing the source code for GreenTE and FGH, respectively.

\section{REFERENCES}

[1] Global Action Plan, "An inefficient truth". http://www.globalactionpaln.rog.uk/, December, 2007.

[2] K. W. Roth, F. Goldstein and J. Kleinman, "Office and telecommunications equipment in commerical buildings-volume I: Energy consumption baseline”. In Technical Report 7289500, Arthur D. Little, Inc., 2002.

[3] A. GreenBerg, J. Hamilton, D. Maltz and P. Patel, "The cost of a cloud: Reserch Problems in Data Centre Networks". In ACM SIGCOMM CCR, 39 (1), January 2009, pp. 68-73.

[4] A. Qureshi, "Cutting the electric bill for Internet-scale systems". In ACM SIGCOMM, Barcelona, Spain, 2009.

[5] J. Y. Yen, "Finding the K Shortest Loopless paths in a network". In Management Science, 17(11), 1971.

[6] J.-P. Vasseur, M. Pickavet, and P. Demeester. Network Recovery: Protection and Restoration of Optical, SONET-SDH, IP, and MPLS, pages 397-422. Morgan Kaufmann Publishers Inc., San Francisco, CA, 2004.
[7] D. Wang and G. Li. Efficient distributed bandwidth management for MPLS fast reroute. IEEE/ACM Trans. Netw., 16(2):486-495, 2008.

[8] V. Sharma and F. Hellstrand. Framework for multi-protocol label switching (MPLS)-based recovery, 2003. IETF RFC 3469.

[9] H. Saito, Y. Miyao, and M. Yoshida. Traffic engineering using multiple multipoint-to-point LSPs. In Proc. of INFOCOM, volume 2, pages 894901, 2000.

[10] S. Soh, S.Rai, "CAREL: Computer Aided Reliablity Evaluator for Distributed Computing Networks", in IEEE Transactions on Parallel and Distributed Systems, Vol. 2, No. 2, April 1991.

[11] R. Miller, Switching Theory, Vol. I: Combinational Circuits. In New York: Wiley, 1965.

[12] I. Ganichev, B. Dai, B. Godfrey, and S. Shenker. YAMR: Yet another multipath routing protocol. SIGCOMM Comput. Commun. Rev., 40(5):14-19, 2010.

[13] W. Zhang, J. Tang, C. Wang, and S. de Soysa, "Reliable adaptive multipath provisioning with bandwidth and differential delay constraints," in Proc. of INFOCOM, pages 2178-2186, 2010.

[14] Y.Wang, H.Wang, A. Mahimkar, R. Alimi, Y. Zhang, L. Qiu, and Y. R. Yang, "R3: Resilient routing reconfiguration". In Proc. of ACM SIGCOMM, pages 291-302, 2010.

[15] M. L. Shooman, Reliability of Computer Systems and Networks. In WILEY, 2002.

[16] Telemark. Telemark survey. http://www.telemarkservices.com/, 2006.

[17] W. Fisher, M. Suchara, and J. Rexford, "Greening backbone networks: reducing energy consumption by shutting off cables in bundled links". In Green Networking, 2010.

[18] M. Zhang, C. Yi, B. Liu, and B. Zhang, "GreenTE: Power-Aware Traffic Engineering". In ICNP, 2010.

[19] “CPLEX". [Online]. Available: http://www-01.ibm.com/software/ integration/optimization/cplex-optimizer/.

[20] S. Uhlig, B. Quoitin, J. Lepropre, and S. Balon, "Providing Public Introdomain Traffic Matrices to the Research Community". ACM SIGCOMM Computer Communication Review, Vol. 36, no. 1, pp. 8386, January 2006.

[21] "Yin Zhang's Abilene TM". http://www.cs.utexas.edu/ yzhang/ research/AbileneTM/.

[22] N. Spring, R. Mahajan, D. Wetherall, and T. Anderson, "Measuring ISP Topologies with Rocketful" In IEEE/ACM Transactions on Networking, vol. 12, no. 1, pp.2-16, 2004.

[23] M. Roughan, A. Greenberg, C. Kalmanek, M. Rumsewicz, J. Yates and Y. Zhang, "Experience in Measuring Backbone Traffic Variability: Models, Metrics, Measurements and Meaning". In ACM SIGCOMM Internet Measurement Workshop, 2002.

[24] M. Suchara, D. Xu, R. Doverspike, D. Johnson and J. Rexford, "Network Architechture for Joint Failure Recovery and Traffic Engineering”, in SIGMETRICS'11, June 7-11, 2011.

[25] D. Applegate, L. Breslau, and E. Cohen, "Coping with network failures: Routing strategies for optimal demand oblivious restoration," in Proc. ACM SIGMETRICS, June 2004.

[26] G. Lin, S. Soh, K. Chin and M. Lazarescu, "Power-Aware Routing in Networks with Delay and Link Utilization Constraints," in LCN, 2012.

[27] G. Lin, S. Soh, K. Chin and M. Lazarescu, "Efficient Heuristics for Energy-Aware Routing in Networks with Bundled Links," accpected by Computer Networks, 2013.

[28] G. Lin, S. Soh, K. Chin and M. Lazarescu, "Energy-Aware Two LinkDisjoint Paths Routing," submitted to HPSR 2013.

[29] E. Osborne and A. Simha, Traffic Engineering with MPLS, Cisco Press, 2002.

[30] L. Chiaraviglio, M. Mellia, and F. Neri, "Minimizing ISP Network Energy Cost: Formulation and Solutions," in IEEE/ACM Transactions on Networking, 2011. 